\title{
A Study of the Surgical Outcome and Impact on Semen Parameters after Laparoscopic Varicocelectomy and Sub Inguinal Varicocelectomy
}

\author{
Bhaskarapprakash A. R. ${ }^{1}$, Velmurugan P. ${ }^{2}$, Chandru T. ${ }^{3}$, Sriram K. ${ }^{4}$, Natarajan K. ${ }^{5}$
}

\begin{abstract}
${ }^{1}$ Department of Urology, Sri Ramachandra Institute of Higher Education and Research, Chennai, Tamilnadu, India. 2Department of Urology, Sri Ramachandra Institute of Higher Education and Research, Chennai, Tamilnadu, India. ${ }^{3}$ Department of Urology, Sri Ramachandra Institute of Higher Education and Research, Chennai, Tamilnadu, India. ${ }^{4}$ Department of Urology, Sri Ramachandra Institute of Higher Education and Research, Chennai, Tamilnadu, India.

${ }^{5}$ Department of Urology, Sri Ramachandra Institute of Higher Education and Research, Chennai, Tamilnadu, India.
\end{abstract}

\section{ABSTRACT}

\section{BACKGROUND}

Varicocele is the dilatation of pampiniform plexuses which can cause infertility. Incidence of varicocele is around $15-80 \%$ and remains the most common treatable cause of infertility in men. The aim of this study was to identify the preferred method of varicocelectomy in patients with infertility, which gives optimal improvement in semen parameters. This study compares laparoscopic varicocelectomy and subinguinal varicocelectomy.

\section{METHODS}

This is a prospective interventional study carried out in the Department of Urology, Sri Ramachandra Institute of Higher Education and Research (SRIHER), Chennai from August 2017 to July 2019. We included patients with documented infertility with clinical varicocele. Only couples with no other attributable causes including female factors for infertility were included. Patients with previous history of surgery for inguinal hernia, testicular pathology were excluded from the study. We included 25 patients who satisfied the inclusion criteria. They were subjected to physical examination, doppler study and semen analysis. The patients were randomised into 2 groups, A \& B by random allocation. Group A (12 patients) underwent laparoscopic varicocelectomy and group B (13 patients) underwent subinguinal varicocelectomy. All patients were regularly followed up at 3 months and 6 months period postsurgery.

\section{RESULTS}

Review at immediate post-operative period, follow ups at $3 \& 6$ months were undertaken. History of conception, clinical examination, semen analysis according to WHO 2010 manual and doppler studies by Sarchetsi scale were performed. Three factors in seminal parameters namely sperm concentration, motility \& morphology were assessed. Varicocele recurrence was also assessed. There had been a statistically significant improvement in semen parameters in all the patients who underwent varicocelectomy ( $\mathrm{p}$ value $\mathrm{p}<0.005$ ). On comparing the laparoscopic and sub-inguinal surgical techniques, there was no significant variation in increase in sperm concentration, sperm motility and morphology.

\section{CONCLUSIONS}

Varicocelectomy improves the semen parameters. No significant variation was observed when the different surgical approaches of varicocelectomy were compared.

\section{KEY WORDS}

Male Infertility, Varicocelectomy, Laparoscopic Varicocelectomy, Sub Inguinal Varicocelectomy, Varicocele
Corresponding Author:

Velmurugan $P$.,

Associate Professor,

Department of Urology,

SRIHER, 1 Ramachandra Nagar,

Porur, Chennai-600116,

Tamilnadu, India.

E-mail:velumsdoc@rediffmail.com

DOI: $10.14260 / \mathrm{jemds} / 2020 / 261$

Financial or Other Competing Interests: None.

How to Cite This Article:

Bhaskarapprakash AR, Velmurugan $P$, Chandru T, et al. A study of the surgical outcome and impact on semen parameters after laparoscopic varicocelectomy and sub inguinal varicocelectomy. J. Evolution Med. Dent. Sci. 2020;9(14):1202-1207, DOI: $10.14260 /$ jemds $/ 2020 / 261$

Submission 20-11-2019,

Peer Review 14-03-2020,

Acceptance 21-03-2020,

Published 06-04-2020.

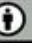




\section{BACKGROUND}

Varicocele is the dilatation of the pampiniform plexuses. The prevalence of varicocele is approximately $15 \%$ in the general population, $19-41 \%$ in men with primary infertility and 45-81 $\%$ in men with secondary infertility.(1) The association of infertility and varicocele was highlighted first by Tulloch in 1952, where he documented that varicocelectomy in azoospermic patients resulted in return of normal semen parameters post operatively and spontaneous pregnancy.(2) The process by which the varicocele causes defective spermatogenesis is still elusive. Various hypotheses have been put forward for decreased spermatogenesis in patients with varicocele. They are oxidative stress, heat stress and testicular venous hypertension leading to hypo perfusion and blood stasis which causes impaired spermatogenesis, DNA damage, decreased sperm motility.(3)

Effect of varicocelectomy has been a matter of debate, but recent studies give a strong recommendation for varicocelectomy in infertile men. One study had reported a $33 \%$ pregnancy rates in patients who underwent surgical varicocelectomy and a $15.5 \%$ pregnancy rates in the controls who had no varicocelectomy.(4) Apart from fertility aspect varicocele repair has proved to decrease the amount of oxidative stress. Even in patients opting for assisted reproductive technology, varicocelectomy prior to ART increases the pregnancy rate and is cost-effective.(5)

The various techniques of repair are as follows-

- Conventional open retroperitoneal- Palomo procedure.

- Laparoscopic varicocelectomy.

- Sub inguinal varicocelectomy.

- Microsurgical sub inguinal- varicocelectomy.

- Radiological procedures- percutaneous embolization of veins.

With the advances in technology and minimal access techniques, laparoscopy has been the preferred approach due to early recovery and better cosmesis. Sub inguinal procedure offers minimal scar and post-operative complications. With the aim of choosing a better surgical technique for restoration of fertility, we have analysed and compared the surgical outcome in terms of its impact on semen parameters postsurgery: laparoscopic varicocelectomy and sub inguinal varicocelectomy.

\section{METHODS}

This is prospective interventional study conducted from August 2017 to July 2019 in patients attending the OPD in the department of Urology, Sri Ramachandra institute for higher education and research, Chennai.

\section{Inclusion Criteria}

- Patients with documented infertility.

- Clinically palpable varicocele.

- Patients with abnormal semen parameters.

- No other attributable causes for infertility.

- No factor for infertility in the female partner.

\section{Exclusion Criteria}

- Previous surgery for inguinal hernia.

- Previous surgery for testicular pathology.

- Congenital abnormalities.

- $\quad$ Prior surgery for varicocele.

\section{Patient Selection}

- All the patients attending the infertility clinic in Department of Urology, Sri Ramachandra institute of higher education and research underwent history elicitation, clinical examination, semen analysis (as per WHO manual 2010) (6)

- Patients with abnormal semen parameters and clinical varicocele were selected.

- Doppler study of the scrotum (presence of varicocele graded using Sarteschi scale)(7) was done for these patients

- The patients fulfilling the selection criteria were counselled for varicocelectomy and consent for the study obtained.

- $\quad$ The patients were randomized into Group A (laparoscopic varicocelectomy) and Group B (sub-inguinal varicocelectomy) by random allocation method.

The laparoscopic varicocelectomy was done by routine 3 transperitoneal ports, 1 in the umbilicus for camera and two working ports in right \& left iliac fossa lateral to inferior epigastric vessels. Peritoneum over the spermatic vessels was incised, veins were identified, ligated with clips and cut, sparing the arteries. Port sites was closed with absorbable sutures and skin with staplers.(8)

In subinguinal varicocelectomy, a small transverse incision is made just inferior to the level of the external ring. Scarpa's fascia is split, cord structures identified using blunt and sharp dissection. Cord is brought up to the level of the skin incision and secured with a vessel tape. With the help of surgical loupe, the cord structures were dissected, veins were identified, ligated with 3-0 silk and divided sparing the arteries and lymphatic channels. Cord was replaced and wound closed with absorbable sutures.(9) The duration of hospital stay, postoperative analgesic requirement was noted for the patients.

Following factors were assessed during follow up-

\section{In the Immediate Post Op Period}

Patients were assessed for pain as per visual analog scale. And examined for complications like hematoma and infection.

\section{3 and 6 Months Post Operatively}

1. History including the history of conception or pregnancy.

2. Clinical examination.

3. Investigations.

a. Semen analysis.

b. Ultrasonography and Doppler study.

\section{Semen Analysis}

We compared the improvement in semen parameters in all patients (combining both groups pre and post operatively), to assess the benefit of varicocele repair. Three vital parameters 
compared were sperm concentration, total motility and sperm morphology.

\section{Statistical Analysis}

The SPSS software (Statistical Package for the Social Sciences, version 13.0, SPSS Inc, Chicago, Illinois, USA) was used to analyse the data. Paired T-test and Wilcoxon signed rank test, Mann Whitney test were used to analyse as the data didn't have the normal distribution. A p value $<0.005$ was considered significant.

\section{RESULTS}

Twenty-five patients were included in the study after they fulfilled the inclusion and exclusion criteria. The demographic parameters of the patients in both the arms were similar. Most of the patients belonged to age group 31-35 years followed by 26-30 years.

\begin{tabular}{|cccc|}
\hline Age of Patients & Laparoscopic No. of Cases & Sub Inguinal & Total \\
21-25 years & 0 & 0 & 0 \\
26-30 years & 6 & 3 & 9 \\
31-35 years & 5 & 6 & 11 \\
36-40 years & 1 & 4 & 5 \\
41-45 years & 0 & 0 & 0 \\
\hline \multicolumn{3}{|c|}{ Table 1. Age Distribution of Patients } \\
\hline
\end{tabular}

Though the prevalence of varicocele is predominant in secondary infertility patients, in our study majority of the patients were with primary infertility $(22 / 25)$. Three were with secondary infertility.

\section{Grading of Varicocele}

On clinical examination, all patients had bilateral varicocele. Nineteen patients had grade II varicocele and 6 patients had grade III varicocele.

\begin{tabular}{|cccc|}
\hline Grading of Varicocele & Laparoscopic Cases & Sub Inguinal & Total \\
Grade 3 & 6 & 7 & 13 \\
Grade 4 & 4 & 3 & 7 \\
Grade 5 & 2 & 3 & 5 \\
\hline Table 2. Doppler Grading as per Sarteschi Grading System \\
\hline
\end{tabular}

There was no significant difference in both the arms when the grading of varicocele was compared both by clinical and by doppler examinations.

\section{Semen Parameters}

Semen analysis was done as per WHO (2010) standards preoperatively. Seventy-two percentage of patients had oligospermia in both arms (18 patients) with a mean sperm concentration of $7.82 \mathrm{million} / \mathrm{ml}$. The rest of them had normal sperm concentration with sub normal motility and morphology. Nine patients had asthenozoospermia in our study. Twenty one of the 25 patients (84\%) had morphologic defects. The patients in both arms had similar seminal parameters before undergoing surgery.

\begin{tabular}{|cccccc|}
\hline & N & Mean & $\begin{array}{c}\text { S.D. } \\
\text { Concentration }\end{array}$ & Std Error Mean & p \\
Laparoscopic & 12 & 16.7 & 12.94 & 3.478 & .614 \\
subinguinal & 13 & 13.48 & 18.473 & 5.123 & \\
& \multicolumn{5}{c}{ Total Motility } \\
Laparoscopic & 12 & 46.58 & 12.94 & 5.623 & .399 \\
subinguinal & 13 & 40.54 & 15.634 & 4.336 & \\
Laparoscopic & 12 & 3.33 & 2.425 & 0.700 & .166 \\
subinguinal & 13 & 2.00 & 2.236 & 0.620 & \\
\hline \multicolumn{7}{|c|}{ Table 3. Distribution of Seminal Parameters in 2 Groups in } \\
Preoperative Status \\
\hline
\end{tabular}

\section{Post-Operative Period}

The following findings were noted.

Immediate postoperative period.

Pain: Pain scale assessment and the analgesic requirements were similar in both groups.

Fever: One patient in group A had fever in the first post op day which subsided on continuation of antibiotics for 2 days and he was discharged on the $3^{\text {rd }}$ post op day.

Wound Infection: One patient developed wound infection in Group B which was evident on the $3^{\text {rd }}$ post day and it required hospital admission and was treated conservatively.

No hematoma was detected in any of the patients.

All patients were followed up regularly at the intervals of 3 months post-surgery and 6 months post- surgery. No significant complication occurred in any of the 25 patients. On Doppler study no patient had recurrence of varicocele.

\section{Semen Analysis during $3^{\text {rd }}$ and $6^{\text {th }}$ Month Follow Up}

We compared the change in semen parameters in terms of 1) An entire cohort of 25 patient's pre and post-operative status and 2) Compared as cohorts undergoing the two surgical approaches.

No significant difference was seen in the macroscopic appearance of the semen post-operatively. Three vital microscopic parameters compared were sperm concentration, total motility and sperm morphology. As the distribution was not normal, T-test and Wilcoxon signed rank test were used for statistical analysis.

\section{Sperm Concentration}

In the preoperative analysis, the mean concentration of sperm in the 25 patients was 15.02 Million/ml. Eighteen patients were oligospermic (concentration levels $<15 \mathrm{mil} / \mathrm{ml}$ ) with a mean sperm concentration of 7.42 million/ ml,

\section{At 3 Months}

The sperm concentration improved in all patients and the mean sperm concentration increased from 15.02 million $/ \mathrm{ml}$ to 18.22 million/ml post-operatively. Among the 18 oligospermic patients, sperm concentration increased in 7 patients $(38.8 \%)$ and the remaining 11 patients (61.2\%) remained oligospermic. Even in this category their mean concentration increased to 9.32 million $/ \mathrm{ml}$. 


\section{At 6 Months}

The sperm concentration further improved in all patients and the mean sperm concentration increased to 22.89 million $/ \mathrm{ml}$. Only 7 patients (38.8\%) remained oligospermic with an increase in mean to 10.57 million/ml.

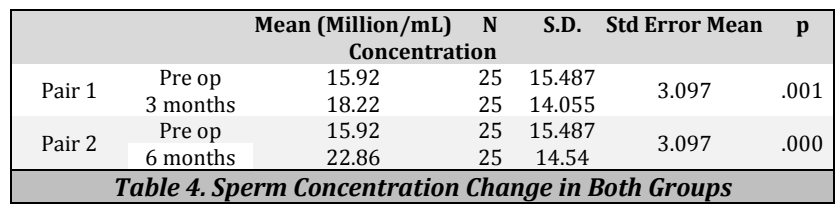

This table shows the increase in sperm concentration was statistically significant $p$ value: preop- 3 months $p=0.001$; pre op -6 months $p=0.000$

\section{Total Motility}

The mean total motility of sperm in the 25 patients was $43.44 \%$. Nine patients (36\%) had asthenozoospermia (total motility $<40 \%$ ) with the mean total motility of this group being $25.2 \%$.

\section{At 3 Months}

The mean total motility increased to $50.96 \%$. In the asthenozoospermic group, 4 (44.44\%) patients had improved motility to normal levels. In the rest of the 5 patients the mean total motility increased to

$34.4 \%$.

\section{At 6 Months}

Further increase in total motility was observed with mean increased to 52.96. Only one patient who had decreased motility remained asthenozoospermic.

\begin{tabular}{|c|c|c|c|c|c|c|}
\hline & & Mean Total Mo & $\begin{array}{l}\mathbf{N} \\
\text { ity \% }\end{array}$ & S.D. & Error Mean & p \\
\hline Pair & Pre op & $43.44 \%$ & 25 & 17.481 & 3.496 & \multirow{2}{*}{.001} \\
\hline 1 & 3 months & $50.96 \%$ & 25 & 11.851 & 2.370 & \\
\hline Pair & Pre op & $43.44 \%$ & 25 & 15.487 & 3.496 & \multirow{2}{*}{.000} \\
\hline 2 & 6 months & $52.96 \%$ & 25 & 14.542 & 2.542 & \\
\hline
\end{tabular}

This table shows the increase in sperm total motility was statistically significant. $p$ value: preop- 3 months $\mathrm{P}=0.001$; pre op -6 months $\mathrm{p}=0.000$.

\section{Morphology}

The mean of morphologically normal sperms in the 25 patients was $2.64 \%$. Around $21(84 \%)$ patients had teratozoospermia (morphologically normal sperms $<4 \%$ ) and the mean of normal sperms in this category was $1.08 \%$.

\section{At 3 Months}

The mean of morphologically normal sperms in the 25 patients at 3 months interval had improved to

$5.24 \%$. Among the 21 patients who had teratozoospermia, in 15 patients the morphology has improved to $>4 \%$ and in the remaining 6 patients the mean percentage of morphology had increased to $3.16 \%$.

\section{At 6 Months}

The mean of morphologically normal sperms in the 25 patients improved at 6 months interval to $8.40 \%$. All patients improved the morphology to normal levels in 6 months.

\begin{tabular}{|c|c|c|c|c|c|c|}
\hline & & Mean & $\begin{array}{r}\mathrm{N} \\
\text { Normal }\end{array}$ & $\begin{array}{l}\text { S.D. } \\
\text { ms }\end{array}$ & Std Error Mean & p \\
\hline \multirow[b]{2}{*}{ Pair 1} & Pre op & $2.64 \%$ & 25 & 2.378 & .476 & \multirow[b]{2}{*}{.000} \\
\hline & 3 months & $5.24 \%$ & 25 & 2.166 & 0.433 & \\
\hline \multirow{2}{*}{ Pair 2} & Pre op & $2.64 \%$ & 25 & 2.378 & 0.476 & \multirow{2}{*}{.000} \\
\hline & 6 months & $52.96 \%$ & 25 & 4.542 & 2.542 & \\
\hline
\end{tabular}

This Table shows the marked increase in morphology and statistically significant $p$ value. Pre-op- 3 months $p=0.000$; pre op -6 months $\mathrm{p}=0.000$.

The observations made in the above study have shown statistically significant improvement in semen parameters in infertile men with varicocele after varicocele repair.

\section{Comparison of the Impact on Semen Parameters Between} These Two Surgical Techniques

\begin{tabular}{|cccc|}
\hline & $\begin{array}{c}\text { Group A- Laparoscopic } \\
\text { Varicocelectomy }\end{array}$ & $\begin{array}{c}\text { Group B- Subinguinal } \\
\text { Varicocelectomy }\end{array}$ & \\
& N=12 & Mean & \\
Pre op & 16.7012 .048 & 13.4818 .473 & 0.201 \\
3 Months & 19.4210 .113 & $17.1217,281$ & 0.156 \\
6 months & 23.8811 .021 & $21.9217,595$ & 0.327 \\
\hline Table 7. Comparison of Sperm Concentration- Group A \& Group B \\
\hline
\end{tabular}

The increase in sperm concentration between two groups, laparoscopic varicocelectomy \& sub inguinal varicocelectomy was not statistically significant in post op period of 3 months and 6 months. Both techniques had a similar enhancement. Pre-op -3 months $P$ value $=0.156$; Pre op -6 months $p$ value $=0.327$.

\begin{tabular}{|cccc|}
\hline & $\begin{array}{c}\text { Group A- Laparoscopic } \\
\text { Varicocelectomy } \\
\text { N=12 }\end{array}$ & $\begin{array}{c}\text { Group B- Subinguinal } \\
\text { Varicocelectomy } \\
\text { N=13 }\end{array}$ & \\
Pre op & Mean S.D. & Mean S.D. & $\mathrm{p}$ \\
3 & $46.58 \% 19.477$ & $40.54 \% 15.634$ & .399 \\
Months & $52.58 \% 13.386$ & $49.46 \% 10.564$ & .522 \\
6 & $52.33 \% 12.478$ & $53.54 \% 13.402$ & .818 \\
months & & & \\
\hline \multicolumn{4}{|c|}{ Table 8. Comparison of Sperm Motility in Group A \& Group B } \\
\hline
\end{tabular}

The increase in the mean total motile sperms between laparoscopic varicocelectomy \& sub inguinal varicocelectomy was not statistically significant. On statistical analysis between Group A laparoscopic varicocelectomy \& Group B sub inguinal varicocelectomy in terms of total motility Preop -3months \& preop -6 months, the P value were $0.522 \& 0.818$ respectively.

\begin{tabular}{|cccc|}
\hline $\begin{array}{c}\text { Group A- Laparoscopic } \\
\text { Varicocelectomy } \\
\text { N=12 }\end{array}$ & $\begin{array}{c}\text { Group B- Subinguinal } \\
\text { Varicocelectomy }\end{array}$ & \\
& Mean S.D. & Mean S.D. & $\mathrm{p}$ \\
Pre op & $3.33 \% 2.45$ & $2.00 \% 2.236$ & .166 \\
3 Months & $5.75 \% 2.094$ & $4.77 \% 2.204$ & .267 \\
6 months & $8.67 \% 3.420$ & $8.15 \% 5.352$ & .776 \\
\hline Table 9. Comparison of Sperm Morphology in Group A \& Group B \\
\hline
\end{tabular}

The increase in the mean of morphologically normal sperms between the two groups Group A laparoscopic varicocelectomy \& group B sub inguinal varicocelectomy was not statistically significant. Group A laparoscopic varicocelectomy \& group B sub inguinal varicocelectomy. Preop - months $p$ value $=0.267$; Pre op -6 months $p$ value $=0.776$. 
The observations made in this study was that varicocele repair has a role in improvement of semen parameters, but no statistically significant difference was observed when the surgical outcome was analysed between the laparoscopic varicocelectomy and sub-inguinal varicocelectomy.

\section{DISCUSSION}

Varicoceles are abnormal dilatations of the pampiniform plexus of veins within the spermatic cord. In certain patients, varicoceles can cause testicular damage resulting in loss of testicular volume, spermatogenic dysfunction, disruption of hormone production, and sperm DNA damage. The pathologic mechanism of testicular dysfunction secondary to varicocele have been attributed to the increased oxidative stress, local hormonal imbalances, stasis of blood (toxin accumulation), testicular hypoperfusion, and heat stress. The exact mechanism by which the surgical correction of varicocele improves fertility in affected men remains unknown. A 2004 Cochrane meta- analysis of randomized studies concluded that varicocele repair in a couple with otherwise unexplained infertility could not be recommended due to lack of significant improvement in semen parameters. Recent RCTs and nonRCTs have demonstrated that varicocele repair is clearly associated with significant improvement in semen parameters as well as spontaneous pregnancy rates compared with nonintervention. ${ }^{(10,11)}$

This significant benefit of varicocelectomy even extends to men with nonobstructive azoospermia. In one meta-analyses of azoospermic patients with varicoceles, Esteves et al. found that varicocelectomy led to return of sperm to the ejaculate in $43.9 \%$ of patients and was associated with a $13.6 \%$ natural spontaneous pregnancy rate. In addition, they found that correction of varicocelectomy in this group was associated with improved sperm retrieval rates (odds ratio [OR] 2.65, 95\% confidence interval [CI] 1.69-4.14; $\mathrm{p}<0.001$ ).(12)

There are various methods for varicocelectomy, but none has been superior in efficacy in relation to treatment of infertility in patients with varicocele. Shamsa et al compared 3 varicocelectomy methods on 3 groups comprising 30 patients each. In this study all had the same response in increasing the semen parameters. When comparing the post-operative complications like hydrocele, recurrence and operative time, sub- inguinal varicocelectomy under LA was found to preferred method.(13) Dr. Haluk So"ylemez et al in his claims that laparoscopic varicocelectomy is better in terms of patient comfort and early return to activity and advices laparoscopic to be gold standard.(14)

Armand Zini et al showed that though technically challenging, microsurgical sub inguinal varicocelectomy has less complications and early return to work when compared to other modalities of varicocele repair.(15) A recent metanalysis provides evidence for improvement of semen parameters following varicocelectomy, but all techniques have similar improvement in semen parameters, except for the advantages in reducing complications post-surgery, with microsurgical approach having least complications.(5) In a recent study, Abdul Mageed compared laparoscopic and sub inguinal varicocelectomy and concluded that both groups had similar impact on semen parameters and complications. ${ }^{(16)}$

In a meta- analysis of 33 studies, conducted in over 5000 patients, various current varicocelectomy techniques were analysed. The surgical outcome and improvement in semen parameters were calculated. They calculated that the overall pregnancy rate was $38.37 \%$ (954/2486). The incidence of recurrence of varicocele were more in radiological procedure when compared with surgical methods (12\% Vs 9.6\%). The increase in seminal parameters varied from 50 to $80 \%$ in operated patients with results more in favour of microsurgical inguinal surgery (17). In a case series, varicocelectomy using loupe-assisted inguinal technique could improve semen parameters and pregnancy rate with a low postoperative complication rate (18). In a review article varicocelectomy before assisted reproduction helps in improving the outcomes, irrespective of the technique of varicocelectomy.(19)

In our study of 25 patients, post operatively there was improvement in semen parameters in both groups with mean sperm concentration increase from 15.02 millon/ml to 22.89 million $/ \mathrm{ml}$. Among the 18 patients in entire cohort who were oligospermic, 11 patients improved their sperm count to normal. The mean total sperm motility increased from 43.44 $\%$ to $52.96 \%$. The mean sperm morphology also improved from $2.64 \%$ to $8.4 \%$. When the semen parameters were compared in the two surgical methods, no statistically significant difference was observed, the improvements were similar in both groups.

The limitations of this study were small sample size, short duration of patient's follow up, lack of information of the impact on pregnancy rates and non-usage of operating microscope.

\section{CONCLUSIONS}

Based on our study, there is a definite improvement in semen parameters with statistically significant increase in sperm concentration, sperm total motility \& sperm morphology following varicocele repair with both laparoscopic varicocelectomy \& sub-inguinal varicocelectomy. There was no difference between laparoscopic varicocelectomy and subinguinal varicocelectomy when surgical outcome and sperm parameters were compared. Post-operative complications by both techniques were minimal and insignificant.

Laparoscopic varicocelectomy does provide better cosmesis but sub-inguinal varicocelectomy is cost effective in low resource setting. There was no statistically significant difference observed when the sperm parameters were compared between both the arms. So, both laparoscopic and sub-inguinal varicocelectomy have same efficacy in terms of improvement in semen parameters post varicocele repair in infertile men with clinically detected varicocele.

\section{REFERENCES}

[1] Pastuszak AW, Wang R. Varicocele and testicular function. Asian J Androl 2015;17(4):659-66. 
[2] Tulloch WS. Varicocele in subfertility: results of treatment. British Medical Journal 1955;2(4935):356-8.

[3] Clavijo RI, Carrasquillo R, Ramasamy R. Varicoceles: prevalence and pathogenesis in adult men. Fertil Steril 2017;108(3):364-9.

[4] Marmar JL, Agarwal A, Prabhakaran S, et al. Re-assessing the value of varicocelectomy as a treatment for male subfertility with a new meta-analysis. Fert Ster 2007;88(3):639-48.

[5] Kohn TP, Kohn JR, Pastuszak AW. Varicocelectomy before assisted reproductive technology: are outcomes improved? Fertil Steril 2017;108(3):385-91.

[6] WHO semen analysis manual 2010.

[7] Sarteschi LM, Paoli R, Bianchini M. Menchini Fabrics GF Lo studio Del varicocele con eco-color Doppler. G Ital Ultrasonologia 1993;4:43-9.

[8] Tu D, Glassberg KI. Laparoscopic varicocelectomy. BJU International 2010;106(7):1094-104.

[9] Abdelrahman SS, Eassa BI. Outcome of loupe-assisted sub-inguinal varicocelectomy in infertile men. Nephrourology Monthly 2012;4(3):535-40.

[10] Ficarra V, Crestani A, Novara G, et al. Varicocele repair for infertility: what is the evidence? Curr Opin Urol 2012;22(6):489-94.

[11] Masson P, Brannigan RE. The varicocele. Urol Clin North Am 2014;41(1):129-44.

[12] Esteves SC, Miyaoka R, Roque M, et al. Outcome of varicocele repair in men with non-obstructive azoospermia: systematic review and meta-analysis. Asian J Androl 2016;18(2):246-53.

[13] Shamsa A, Mohammadi L, Abolbashari M, et al. Comparison of open and laparoscopic varicocelectomies in terms of operative time, sperm parameters and complications. Urol J 2009;6(3):170-5.

[14] Soylemez H, Penbegul N, Atar M, et al. Comparison of laparoscopic and microscopic subinguinal varicocelectomy in terms of postoperative scrotal pain. JSLS 2012;16(2):212-7.

[15] Zini A. Varicocelectomy: microsurgical subinguinal technique is the treatment of choice. Can Urol Assoc J 2007;1(3):273-6.

[16] Abdulmageed MU, Al-Azzawi IS. A comparative study between laparoscopic varicocelectomy and open varicocelectomy in a group of Iraqi patients. J Pak Med Assoc 2019;69(Suppl 3)(8):S73-S7.

[17] Diegidio P, Jhaveri JK, Ghannam S, et al. Review of current varicocelectomy techniques and their outcomes. BJU Int 2011;108(7):1157-72.

[18] Shafi H, Delavar MA. The impact of loupe-assisted inguinal varicocelectomy on semen quality and pregnancy rate. Acta Endocrinol (Buchar) 2018;14(2):252-4.

[19] Reddy SVK, Shaik AB, Sailaja S, et al. Outcome of varicocelectomy with different degrees of clinical varicocele in infertile male. Article ID 432950, Advances in Andrology 2015;2015:1. 